\title{
Globalized Power Aware Routing Protocols For Mobile Ad Hoc Networks - Performance Comparison
}

\author{
Humaira Nishat ${ }^{1}$ and Dr.D.Srinivasa Rao ${ }^{2}$ \\ ${ }^{1}$ CVR College of Engineering, Department of ECE., Ibrahimpatan, R.R.District, A.P., India \\ Email: huma_nisha@yahoo.com \\ ${ }^{2}$ Jawaharlal Nehru Technological University Hyderabad, Department of ECE., Hyderabad, India \\ Email: dsraoece@jntuh.ac.in
}

\begin{abstract}
A mobile ad hoc network consists of nodes which are constrained by limited battery power for their operation. Thus, battery management is an important issue in such networks. There are few power aware routing protocols developed for ad hoc wireless networks at data link, network and higher layers to solve the problem of energy management. In this paper, we compare the performance of two globalized power aware routing protocols- minimum battery cost routing (MBCR) and min-max battery cost routing (MMBCR) by comparing the route failure times of both protocols. We have used ns2 to carry out the simulation. From the simulation results we observe that $M M B C R$ have longer network lifetime than MBCR, thus MMBCR performs better than MBCR in a battery constraint mobile ad hoc network environment.
\end{abstract}

Index Terms-MANETS, routing protocols, MBCR, MMBCR, network lifetime.

\section{INTRODUCTION}

Mobile ad hoc network (MANET) [1] is a collection of mobile devices called nodes that communicate with each other over multi-hop wireless links in a collaborative manner without the use of any centralized infrastructure. Due to the high dynamic topology routing protocols used in wired networks cannot be applied to ad hoc networks. A number of routing protocols have been proposed for ad hoc networks. Ad hoc wireless routing protocols are broadly classified into two major categories as either proactive or tabledriven and reactive or on-demand routing protocols. Table driven routing protocols maintains network topology information in the form of routing tables by periodically exchanging routing information. On the other hand, reactive or on-demand routing protocols obtain the necessary routing information by using a connection establishment process.
Nodes in an ad hoc wireless network are constrained by limited battery power and thus, energy management [2] is an important issue in such networks. Few energy efficient routing protocols [3] at the network layer have been proposed in literature to solve the problem of energy management. In this paper, we study the performance of two globalised power aware routing protocols-MBCR \& MMBCR [4] by implementing the two routing algorithms on the same network scenario and comparing the route failure time in each case. The rest of the paper is organized as follows. Section two gives a brief introduction of the two routing protocolsMBCR \& MMBCR. Section three gives the simulation setup. Section four presents the results in the form of performance comparison of the two routing protocols and finally section five concludes the paper.

\section{Description Of The Two Routing Protocols}

Distributing the power consumption evenly among nodes and minimizing the overall transmission power are two difficult tasks in a mobile ad hoc network. Routing algorithms described in [4], [5] attempts to find a balance between these two factors by using node metrics for the route selection process. The authors in [6] proposed a power optimal scheduling and routing protocol which tries to minimize the total average power in the network. In [2], the authors propose a common power protocol (COMPOW) that attempts to satisfy three major objectives: increasing the lifetime of all the nodes, increasing the traffic-carrying capacity of the network and reducing the contention of nodes. In [7], authors proved that COMPOW protocols works well only in a network with a homogeneous distribution of nodes and exists as a special case of the CLUSTERPOW protocol proposed by them. In [8], authors propose a centralized algorithm that calculates the minimum power level for each node that is required to maintain network connectivity based on the global information from all the nodes. The lifetime of mobile 
ad hoc networks depends mainly on each nodes battery capacity. Hence, routing protocols must provide energy efficient route discovery and maintenance mechanisms. The following is a description of two globalized power aware routing protocols MBCR and MMBCR.

\section{A. Minimum Battery Cost Routing (MBCR)}

In this routing algorithm, the path containing nodes with less remaining battery capacity is not selected and thus the algorithm considers the summation of battery charges of all nodes while selecting the path. If the battery cost at any instant of time $t$ is denoted by $c_{i}^{t}$, then the remaining battery capacity of the node is given by

$$
f_{i}\left(c_{i}^{t}\right)=1 / c_{i}^{t}
$$

Where $\mathrm{f}\left(\mathrm{c}_{\mathrm{i}}^{\mathrm{t}}\right)$ represents the battery cost function of host $\mathrm{n}_{\mathrm{i}}$. The higher the value of the function $f_{i}$, the more unwilling is the node to participate in the route selection algorithm. To find a route with the maximum remaining battery capacity, we should select a route $i$ that has the minimum battery cost.

$$
R_{i}=\min \left(R_{j}\right) \text {, for all jeA }
$$

Here $A$ is the set of all routes from source to destination. The disadvantage of MBCR is that since only the summation of values of battery cost functions is considered, there is a little possibility that a route containing nodes with little remaining battery capacity may still be selected.

\section{B. Min-Max Battery Cost Routing (MMBCR)}

In this routing algorithm, the route selection is done based on the battery capacity of all the individual nodes i.e., the battery of each host is used more fairly than in the MBCR protocol. Battery cost $R_{j}$ for route $j$ is defined as

$$
\mathrm{R}_{\mathrm{j}}=\operatorname{Max}_{\mathrm{iEroutej}} \mathrm{f}_{\mathrm{i}}\left(\mathrm{c}_{\mathrm{i}}^{\mathrm{t}}\right)
$$

The desired route $\mathrm{i}$ can be obtained from the equation

$$
\mathrm{R}_{\mathrm{i}}=\operatorname{Min}\left(\mathrm{R}_{\mathrm{j}}, \mathrm{jE} \mathrm{A}\right)
$$

Where $\mathrm{A}$ is the set containing all possible routes. A variant of this routing protocol minimizes the maximum cost after routing $\mathrm{N}$ packets to the destination or after a time period of $t$ seconds.

\section{Simulation SETUP}

We have used network simulator (NS 2.34) for the simulation. NS2 [9], [10] is a discrete event driven simulator developed at the University of Berkeley and the Virtual Inter Network Testbed (VINT) project 1997. We have used Fedora Version 8 as operating system. NS2 is suitable for designing new protocols, comparing different protocols and for traffic evaluations. It is an object oriented simulator written in $\mathrm{C}++$, with OTCL interpreter as a frontend.

The parameters used for carrying out simulation are summarized in table1. The goal of our simulation is to evaluate the network lifetime for both the routing protocol MBCR and MMBCR by considering the energy of nodes during transmission.

TABLE I.

\begin{tabular}{|l|c|}
\hline Parameter & Value \\
\hline Routing Protocols & MBCR \& MMBCR \\
\hline MAC Layer & 802.11 \\
\hline Terrain Size & $500 \mathrm{~m} * 500 \mathrm{~m}$ \\
\hline No. of Nodes & 7 \\
\hline Packet Size & $512 \mathrm{~B}$ \\
\hline Initial Energy & 2 Joules \\
\hline Simulation Time & $60 \mathrm{sec}$ \\
\hline Traffic Source & UDP \\
\hline
\end{tabular}

\section{Simulation RESUlts}

We have created a network scenario of 7 nodes and each node is assigned an initial energy of 2 joules. The simulation time was set to 60 seconds and we have used UDP as the traffic source. There are three different paths from source 0 to destination 4 as shown in the figure 1. Initially, we made node 5 to transmit data to node 1 , node 2 and node 3 and thus node 5 has only 0.5 joules of energy remaining with it. To transmit data from 0 to 4 MBCR selects the route 0-4-5 with maximum battery capacity on an average or the minimum total cost function. The main drawback of this algorithm is that it does not consider the energy of individual nodes resulting in less network lifetime even if a single node in that route dies out due to low battery capacity. Node 5 has less battery capacity and after certain time period it dies out resulting in route failure. MMBCR on the other hand, considers the individual battery capacities of each node and thus selects route 0 1-6-4 for data transmission. In case of MMBCR, the route containing nodes with minimum battery capacity is avoided and route with better battery capacity of nodes is selected resulting in delaying the route failure time and thereby increasing the lifetime of the network. From our simulation results we observe that the route failure time of MBCR is much less than that of MMBCR for the same network scenario. Figure2 gives a comparison of route failure times of MBCR and MMBCR. The route selected for transmission of data packets dies out at 50 seconds in case of MBCR whereas for MMBCR the network failure time is 58.4 seconds. 


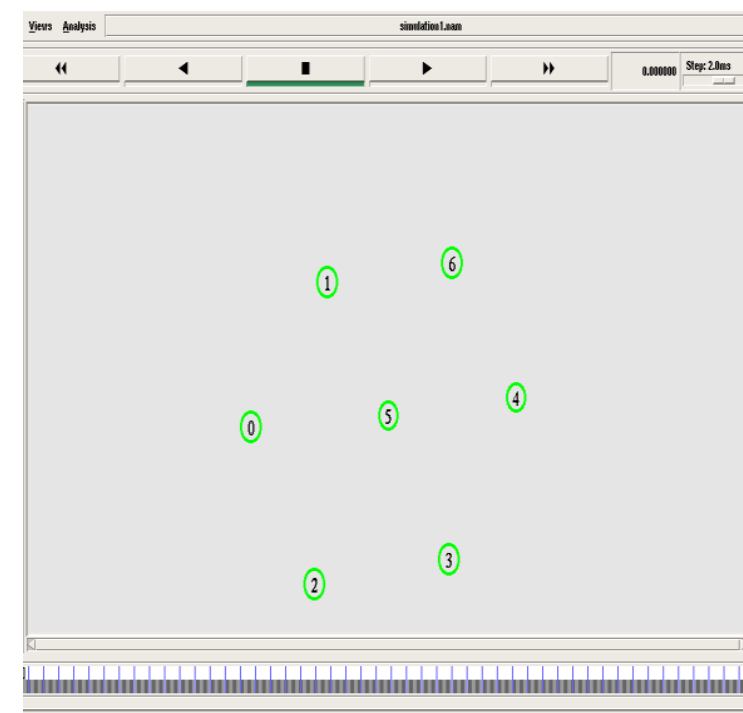

Figure 1. Screenshot showing the network scenario of seven nodes.

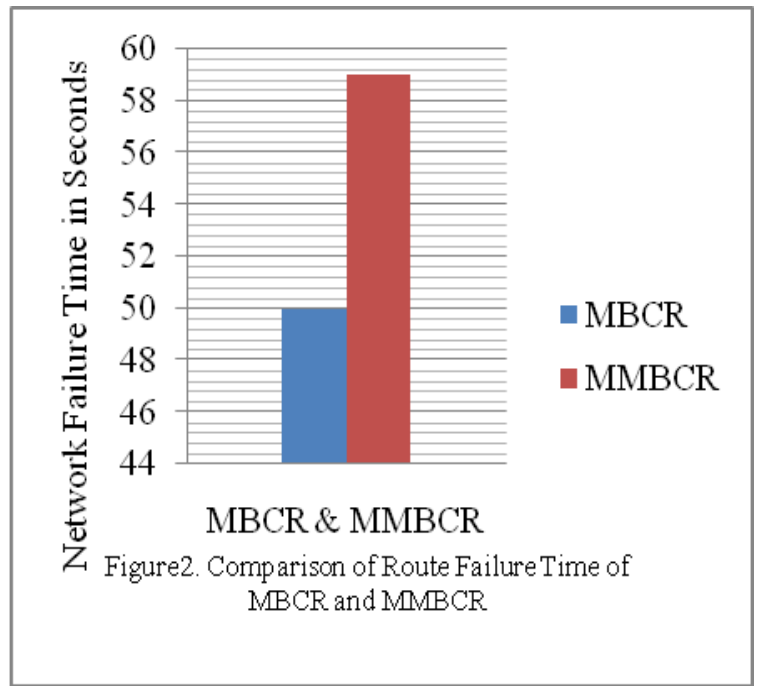

\section{CONCLUSIONS}

From the simulation results we conclude that when we compare the two routing protocols MBCR \& MMBCR in terms of network lifetime, MMBCR performs better than MBCR giving more network lifetime. The main disadvantage of MBCR is that it does not consider the individual node energies though it takes into account the overall battery capacity of the route selected whereas MMBCR selects the route by considering the individual battery capacities of each node in that route thereby increasing the lifetime of network.

\section{REFERENCES}

[1] B.S.Manoj and C. Siva Ram Murthy, "Ad Hoc Wireless Networks: Issues and Challenges," Technical Report, Department of Computer Science and Engineering, Indian Institute of Technology, Madras, India, November 2003.

[2] C.F.Chiasserini and R.R.Rao, "Energy-Efficient Battery Management", Proceedings of IEEE INFOCOM 2000, vol.2, pp-396-403, March 2000.

[3] M.Adamou and S.Sarkar, "A Framework for Optimum Battery Management for Wireless Nodes", Proceedings of IEEE INFOCOM 2002, pp1783-1792, June 2002.

[4] C.K.Toh, "Maximum Battery Life Routing to Support Ubiquitous Mobile Computing in Wireless Ad Hoc Networks", IEEE Communications Magazine, June 2001.

[5] M.Woo, S.Singh and C.S.Raghavendra, "PowerAware Routing in Mobile Ad Hoc Networks", Proceedings of IEEE MOBICOM 2003, pp. 122133, September 2003.

[6] R.L.Cruz and A.R.Santhanam, "Optimal Routing, Link Scheduling and Power Control in Multi-Hop Wireless Networks," Proceedings of INFOCOM 2003, Vol.1 pp.702-711, April 2003.

[7] V.Kawadia, S.Narayanaswamy, R.Rozovsky, R.S.Sreenivas and P.R.Kumar, "Protocols for Media Access Control and power Control in Wireless Networks", Proceedings of IEEE Conference on Decision and Control 2001, Vol.2, pp.1935-1940, December 2001.

[8] V.Kawadia and P.R.Kumar, "Power Control and Clustering in Ad Hoc Networks," Proceedings of IEEE INFOCOM 2003, Vol. 1, pp.459-469, April 2003.

[9] UCB/LBNI/VINT Network Simulator http://www.mash.cs.berkeley.edu/ns/referred on March 2010.

[10] "The Network Simulator NS-2", available at http://www.isi.edu/nsnam/ns/referred on March 2010. 